\title{
ON DUAL SERIES RELATIONS INVOLVING SERIES OF GENERALIZED BATEMAN $K$-FUNCTIONS
}

\section{K. N. SRIVASTAVA}

1. Here, the solution of the dual series relations

$$
\begin{aligned}
\sum_{n=0}^{\infty}\left\{A_{n} / \Gamma(n+l+1)\right\} K_{2 n}^{2 l}(x)=f_{1}(x), & 0 \leqq x<y, \\
\sum_{n=0}^{\infty}\left\{A_{n} / \Gamma(n+l+(1 / 2))\right\} K_{2 n}^{2 l}(x) & =f_{2}(x), \\
y & <x \leqq \infty, l>(-1 / 2)
\end{aligned}
$$

where $K_{2 n}^{2 l}(x)$ is then a generalized Bateman $K$-function, the functions $f_{1}(x), f_{2}(x)$ are prescribed, is obtained by reducing the problem of finding the coefficients $A_{n}$ to that of solving an Abel integral equation.

In recent years, dual series relations involving Fourier-Bessel, Dini series, trigonometric series and series of Jacobi polynomials have been investigated by various workers [1], [2], [5] to [12]. Nere we shall use the method developed by Sneddon and Srivastav for obtaining the solution of dual series relations involving series of generalized Bateman $K$-functions.

As suggested by Sneddon and Srivastav, with a view to simplify the calculations, we shall consider the problem in two stages. First we assume that $f_{2}(x) \equiv 0$ and secondly that $f_{1}(x) \equiv 0$. The solution of the general problem is obtained by adding the two solutions.

2. In this section we list some results for ready reference. Chakrabarty [3] has defined the generalized Bateman $K$-function as follows.

$$
\begin{aligned}
e^{-x / 2} K_{2 n}^{2 l}(x / 2) & =\frac{(-)^{n-l-1}}{\Gamma(n+l+1)}\left\{\frac{d}{d x}\right\}^{n-l-1}\left\{e^{-x} x^{n+l}\right\}, \quad n \geqq l+1 \\
e^{x} K_{2 n}^{2 l}(x) & =\frac{(-)^{n-l-1}}{\Gamma(2 l+2)}(2 x)^{2 l+1}{ }_{1} F_{1}[-n+l+1 ; 2 l+2 ; 2 x]
\end{aligned}
$$

A slight modification leads to the equations

$$
\begin{aligned}
e^{-x / 2} & K_{2(n+\alpha)}^{2(l+\alpha)}(x / 2) \\
& =\frac{(-)^{n-l-1}}{\Gamma(n+l+2 \alpha+1)}\left\{\frac{d}{d x}\right\}^{n-l-1}\left\{e^{-x} x^{n+l+2 \alpha}\right\}, \quad n \geqq l+1,
\end{aligned}
$$

Received by the editors December 13, 1965. 


$$
\begin{aligned}
& e^{x} K_{2(n+\alpha)}^{2(l+\alpha)}(x) \\
& \quad=\frac{(-)^{n-l-1}}{\Gamma(2 l+2 \alpha+2)}(2 x)^{2 l+2 \alpha+1}{ }_{1} F_{1}[-n+l+1 ; 2 l+2 \alpha+2 ; 2 x] .
\end{aligned}
$$

With the help of (2.3) and (2.4) it is easy to prove that

$$
\begin{gathered}
\int_{0}^{\infty} x^{-(2 l+2 \alpha+1)} K_{2(n+\alpha)}^{2(l+\alpha)}(x) K_{2(m+\alpha)}^{2(l+\alpha)}(x) d x \\
=2^{2 l+2 \alpha} \frac{\Gamma(n-l)}{\Gamma(n+l+2 \alpha+1)} \delta_{m n} .
\end{gathered}
$$

Two particular cases of (2.5) are

$$
\begin{aligned}
\int_{0}^{\infty} x^{-2 l-1} K_{2 n}^{2 l}(x) K_{2 m}^{2 l}(x) d x & =2^{2 l} \frac{\Gamma(n-l)}{\Gamma(n+l+1)} \delta_{m n}, \\
\int_{0}^{\infty} x^{-2 l} K_{2 n-1}^{2 l-1}(x) K_{2 m-1}^{2 l-1}(x) d x & =2^{2 l-1} \frac{\Gamma(n-l)}{\Gamma(n+l)} \delta_{m n},
\end{aligned}
$$

where $\delta_{m n}$ is the Kronecker delta. The following results are easily derived from the more general results given in $[4$, p. $293(5)$, p. 405 (20) ]. For $l>(-1 / 2)$ we have

$$
\int_{0}^{x}(x-y)^{-1 / 2} e^{y} K_{2 n}^{2 l}(y) d y=\left\{\Gamma(1 / 2) / 2^{1 / 2}\right\} e^{x} K_{2 n+(1 / 2)}^{2 l+(1 / 2)}(x),
$$

$$
\int_{0}^{x}(x-y)^{-1 / 2} e^{y} K_{2 n-1}^{2 l-1}(y) d y=\left\{\Gamma(1 / 2) / 2^{1 / 2}\right\} e^{x} K_{2 n-(1 / 2)}^{2 l-(1 / 2)}(x),
$$

$$
\int_{x}^{\infty}(y-x)^{-1 / 2} e^{-y} y^{-l-1} K_{2 n}^{2 l}(y) d y
$$

$$
=\left\{\Gamma(1 / 2) / 2^{1 / 4}\right\} \frac{\Gamma(n+l+1 / 2)}{\Gamma(n+l+1)} x^{-l-(3 / 4)} e^{-x} K_{2 n-(1 / 2)}^{2 l-(1 / 2)}(x),
$$

$$
\int_{x}^{\infty} e^{-y} y^{-l-1} K_{2 n}^{2 l}(y) d y=\left\{2^{1 / 2}(n+l)\right\}^{-1} x^{-l-(1 / 2)} e^{-x} K_{2 n-1}^{2 l-1}(x) .
$$

We also require the result

$$
\frac{d}{d x}\left\{e^{x} K_{2 n}^{2 l}(x)\right\}=2 e^{x} K_{2 n-1}^{2 l-1}(x)
$$

of Chakrabarty [3].

We note that if $f(x)$ is continuously differentiable then the Abel in tegral equation 


$$
f(x)=\int_{0}^{x} \frac{\phi(y)}{(x-y)^{1 / 2}} d y
$$

has a continuous solution

$$
\phi(y)=\frac{1}{\pi} \frac{d}{d y} \int_{0}^{y} \frac{f(x)}{(y-x)^{1 / 2}} d x .
$$

Furthermore, if $f(x)$ is continuously differentiable in the interval $[1, \infty)$ then the integral equation

$$
f(x)=\int_{x}^{\infty} \frac{\phi(y)}{(y-x)^{1 / 2}} d y
$$

has a continuous solution given by the equation

$$
\phi(y)=-\frac{1}{\pi} \frac{d}{d y} \int_{y}^{\infty} \frac{f(x)}{(x-y)^{1 / 2}} d x .
$$

This can easily be established by simple methods (cf. [13, p. 229]). The analysis throughout this paper is purely formal and no attempt is made to justify the interchange of various limiting processes.

3. Case I. $f_{2}(x) \equiv 0$. The dual series equations assume the form

$$
\sum_{n=0}^{\infty}\left\{A_{n} / \Gamma(n+l+1)\right\} K_{2 n}^{2 l}(x)=f_{1}(x), \quad 0 \leqq x<y,
$$

$$
\sum_{n=0}^{\infty}\left\{A_{n} / \Gamma(n+l+(1 / 2))\right\} K_{2 n}^{2 l}(x)=0, y<x \leqq \infty, l>-(1 / 2)
$$

Let us suppose that for $0 \leqq x<y$

$$
\begin{aligned}
\sum_{n=0}^{\infty}\left\{A_{n} / \Gamma(n\right. & +l+(1 / 2))\} K_{2 n}^{2 l}(x) \\
= & -\frac{e^{x} x^{2 l+1}}{2^{3 / 4}} \frac{d}{d x} \int_{x}^{y} \frac{g_{1}(u)}{(u-x)^{1 / 2}} d u .
\end{aligned}
$$

Using the orthogonal property (2.6), it can be shown that

$$
\begin{aligned}
& A_{n}=-\frac{\Gamma(n+l+(1 / 2) \Gamma(n+l+1)}{2^{2 l+(3 / 4)} \Gamma(n-l)} \\
& \cdot \int_{0}^{y} e^{x} K_{2 n}^{2 l}(x)\left(\frac{d}{d x} \int_{x}^{y} \frac{g_{1}(u)}{(u-x)^{1 / 2}} d u\right) d x .
\end{aligned}
$$


Since

(3.5) $-\frac{d}{d x} \int_{x}^{y} \frac{g_{1}(u)}{(u-x)^{1 / 2}} d u=\frac{g_{1}(y)}{(y-x)^{1 / 2}}-\int_{x}^{y} \frac{\frac{d}{d u}\left\{g_{1}(u)\right\}}{(u-x)^{1 / 2}} d u$.

We obtain with the help of equations (2.8) and (2.12), the equation

$$
\begin{array}{r}
A_{n}=\frac{\Gamma(n+l+(1 / 2)) \Gamma(n+l+1) \Gamma(1 / 2)}{\Gamma(n-l) \cdot 2^{2 l+(1 / 4)}} \int_{0}^{u} e^{u} K_{2 n-(1 / 2)}^{2 l-(1 / 2)}(u) g_{1}(u) d u, \\
n=0,1,2 \ldots .
\end{array}
$$

If in equation (3.1), we substitute the coefficients $A_{n}$ from (3.6) we get, on interchanging the order of summation and integration

$$
f_{1}(x)=\int_{0}^{y} e^{u} g_{1}(u) K_{1}(u, x) d u, \quad 0 \leqq x<y,
$$

where

$$
K_{1}(u, x)=\sum_{n=0}^{\infty} \frac{\Gamma(n+l+1) \cdot \Gamma(1 / 2)}{\Gamma(n-l) \cdot 2^{2 l+(1 / 4)}} K_{2 n-(1 / 2)}^{2 l-(1 / 2)}(u) K_{2 n}^{2 l}(x) .
$$

With the help of (2.6) and (2.10) it can be shown that

$$
K_{1}(u, x)=e^{u} \cdot u^{l+(3 / 4)} \cdot e^{-x} \cdot x^{l}(x-u)^{-1 / 2} H(x-u),
$$

where $H(t)$ is Heaviside's unit function. Thus the equation (3.7) is equivalent to

$$
e^{x} x^{-l} f_{1}(x)=\int_{0}^{x} \frac{e^{2 u} u^{l+(3 / 4)} g_{1}(u)}{(x-u)^{1 / 2}} d u, \quad 0 \leqq x<y .
$$

This is an Abel integral equation and its solution is

$$
e^{2 u} u^{l+(3 / 4)} g_{1}(u)=\frac{1}{\pi} \frac{d}{d u} \int_{0}^{u} \frac{e^{x} x^{-l} f_{1}(x)}{(u-x)^{1 / 2}} d x .
$$

The coefficients $A_{n}$ may now be computed from relations (3.6) and (3.11).

4. Case II. $f_{1}(x) \equiv 0$. For finding the coefficients satisfying the relations

$$
\sum_{n=0}^{\infty}\left\{A_{n} / \Gamma(n+l+1)\right\} K_{2 n}^{2 l}(x)=0, \quad 0 \leqq x<y,
$$




$$
\begin{aligned}
\sum_{n=0}^{\infty}\left\{A_{n} / \Gamma(n+l+(1 / 2))\right\} K_{2 n}^{2 l}(x)= & f_{2}(x), \\
& y<x \leqq \infty,(l>-(1 / 2),
\end{aligned}
$$

we begin with the assumption that

$$
\begin{aligned}
\sum_{n=0}^{\infty}\left\{A_{n} / \Gamma(n+l+1)\right\} K_{2 n}^{2 l}(x)=2^{5 / 4} e^{-x} x^{l} \int_{y}^{x} \frac{g_{2}(u)}{(x-u)^{1 / 2}} d u \\
\quad \text { for } y<x \leqq \infty .
\end{aligned}
$$

This is equivalent to assuming that

$$
\begin{gathered}
A_{n}=\frac{\Gamma(n+l+1) \Gamma(n+l+(1 / 2)) \Gamma(1 / 2)}{2^{2 l-1} \Gamma(n-l)} \\
\cdot \int_{y}^{\infty} g_{2}(u) u^{-l-(3 / 4)} e^{-u} K_{2 n-(1 / 2)}^{2 l-(1 / 2)}(u) d u .
\end{gathered}
$$

If we multiply both the sides of equation (4.2) by $x^{-l-1} e^{-x}$ and integrate between the limits $x$ to $\infty, y<x \leqq \infty$ we obtain with the help of (2.11) the relation

$$
\begin{aligned}
& \sum_{n=0}^{\infty}\left\{A_{n} / 2^{1 / 2}(n\right.+l) \Gamma(n+l+(1 / 2))\} x^{-l-(1 / 2)} e^{-x} K_{2 n-1}^{2 l-1}(x) \\
&=\int_{x}^{\infty} x^{-l-1} e^{-x} f_{2}(x) d x .
\end{aligned}
$$

Substituting the values of the coefficients $A_{n}$ from (4.4) in equation (4.5) we find on interchanging the order of summation and integration that

$$
x^{l+(1 / 2)} e^{x} F_{2}(x)=\int_{y}^{\infty} g_{2}(u) u^{-l-(3 / 4)} e^{-u} K_{2}(u, x) d u, \quad y<x \leqq \infty,
$$

where

$$
K_{2}(u, x)=\sum_{n=0}^{\infty} \frac{\Gamma(n+l) \Gamma(1 / 2)}{2^{2 l-1 / 2} \Gamma(n-l)} K_{2 n-(1 / 2)}^{2 l-(1 / 2)}(u) \cdot K_{2 n-1}^{2 l-1}(x)
$$

and

$$
F_{2}(x)=\int_{x}^{\infty} e^{-x} x^{-l-1} f_{2}(x) d x
$$

From (2.7) and (2.9) it easily follows that

$$
K_{2}(u, x)=x^{2 l} e^{x-u}(u-x)^{-1 / 2} H(u-x) .
$$


Consequently the equation (4.6) reduces to the integral equation $(4.10) \quad x^{(1 / 2)-l} F_{2}(x)=\int_{x}^{\infty} \frac{e^{-2 u} u^{-l-(3 / 4)}}{(u-x)^{1 / 2}} g_{2}(u) d u, \quad y<x \leqq \infty$.

The solution of this integral equation is

$$
g_{2}(u)=-\frac{e^{2 u} u^{l+(3 / 4)}}{\pi} \frac{d}{d u} \int_{u}^{\infty} \frac{x^{(1 / 2)-l} F_{2}(x)}{(x-u)^{1 / 2}} d x .
$$

The coefficients $A_{n}$ are given by the relations (4.4) and (4.11).

5. Added in proof. The method given above, however, involves sophisticated assumptions and intricate calculation. In this section a much simpler method of solving the problem discussed above is given.

Multiply (1.1) by $e^{x}$, differentiate with respects to $x$, use (2.12) to obtain

$$
\begin{gathered}
\sum_{n=0}^{\infty}\left\{A_{n} / \Gamma(n+l+1)\right\} K_{2 n-1}^{2 l-1}(x) \cdot e^{x} \\
=\frac{1}{2} \frac{d}{d x}\left\{e^{x} f_{1}(n)\right\}, \quad 0<x \leqq y .
\end{gathered}
$$

By using equations (2.9) and (2.10), we can write the equations (5.1) and (1.2) in the form

$$
\begin{aligned}
F_{1}(u) & =\frac{e^{-u}}{(2 \pi)^{1 / 2}} \int_{0}^{u}(u-x)^{-1 / 2} \frac{d}{d x}\left\{e^{x} f_{1}(x)\right\} d x \\
& =\sum_{n=0}^{\infty}\left\{A_{n} / \Gamma(n+l+1)\right\} K_{2 n-1 / 2}^{2 l-1 / 2}(u), \quad 0 \leqq u<y, \\
F_{2}(u) & =\frac{2^{1 / 4}}{\Gamma\left(\frac{1}{2}\right)} u^{l+3 / 4} e^{u} \int_{u}^{\infty}(x-u)^{-1 / 2} e^{-x} x^{-l-1} f_{2}(x) d x \\
& =\sum_{n=0}^{\infty}\left\{A_{n} / \Gamma(n+l+1)\right\} K_{2 n-1 / 2}^{2 l-1 / 2}(u), \quad y<u \leqq \infty
\end{aligned}
$$

The orthogonal property (2.5) gives the coefficients $A_{n}$ which are calculated from the equation 


$$
\begin{aligned}
A_{n}= & \frac{\Gamma(n+l+1) \Gamma\left(n+l+\frac{1}{2}\right)}{2^{2 l-1 / 2} \Gamma(n-l)}\left[\int_{0}^{y} u^{-2 l-1 / 2} K_{2 n-1 / 2}^{2 l-1 / 2}(u) F_{1}(u) d u\right. \\
& \left.+\int_{y}^{\infty} u^{-2 l-1 / 2} K_{2 n-1 / 2}^{2 l-1 / 2}(u) F_{2}(u) d u\right]
\end{aligned}
$$

\section{REFERENCES}

1. W. D. Collins, On dual series equations and their applications to electrostatic problems of spheroidal caps, Proc. Cambridge Philos. Soc. 57 (1961), 367-384.

2. J. C. Cooke and C. J. Tranter, Dual Fourier-Bessel series, Quart. J. Mech. 12 (1959), 379-384.

3. N. K. Charkrabarty, On generalization of Bateman K-function, Bull. Calcutta Math. Soc. 45 (1953), 1-7.

4. A. Erdelyi (Editor), Tables of integral transforms, Vol. 2, McGraw-Hill, New York, 1954.

5. B. Noble, Some dual series equations involving Jacobi polynomials, Proc. Cambridge Philos. Soc. 59 (1963), 363-372.

6. I. N. Sneddon and R. P. Srivastav, Dual series relations. I, Dual relations involving Fourier-Bessel series, Proc. Roy. Soc. Edinburgh Sect. A 66 (1964), 150-160.

7. R. P. Srivastav, Dual series relations. II, Dual relations involving Dini series, Proc. Roy. Soc. Edinburgh Sect. A 66 (1964), 161-172.

8. - Dual series relations. III, Dual relations involving trigonometric series, Proc. Roy. Soc. Edinburgh Sect. A 66 (1964), 173-184.

9. - Dual series relations. IV, Dual relations involving series of Jacobi polynomials, Proc. Roy. Soc. Edinburgh Sect. A 66 (1964), 185-191.

10. C. J. Tranter, Dual trigonometric series, Proc. Glasgow Math. Assoc. 4 (1959), 49-57.

11. - A further note on dual trigonometric series, Proc. Glasgow Math. Assoc. 4 (1960), 198-200.

12. - An improved method for dual trigonometrical series, Proc. Glasgow Math. Assoc. 6 (1964), 136-140. 1922.

13. E. T. Whittaker and G. N. Watson, $A$ course of modern analysis, Cambridge,

M. A. College of Technology, Bhopal (M.P.) India 